\title{
Low Gestational Weight Gain in Obese Women and Pregnancy Outcomes
}

\author{
Milene Moehlecke ${ }^{1}$ Fabíola Costenaro ${ }^{1}$ Angela AJ Reichelt ${ }^{2}$ Maria Lúcia R. Oppermann ${ }^{3}$ \\ Cristiane B. Leitão ${ }^{1,2}$ \\ ${ }^{1}$ Department of Endocrinology, Universidade Federal do Rio Grande \\ do Sul (UFRGS), Porto Alegre, Brazil \\ 2 Endocrine Division, Hospital de Clínicas de Porto Alegre (HCPA), Rio \\ Grande do Sul, Brazil \\ ${ }^{3}$ Department of Gynecology and Obstetrics, Hospital de Clínicas de \\ Porto Alegre, Rio Grande do Sul, Brazil \\ Address for correspondence Milene Moehlecke, Endocrine Division, \\ Hospital de Clínicas de Porto Alegre, Universidade Federal do Rio \\ Grande do Sul, Rua Ramiro Barcelos, 2350/Prédio 12, $4^{\circ}$ andar, CEP: \\ 90035-003 Porto Alegre, RS, Brazil \\ (e-mail: milenemoehlecke@yahoo.com.br).
}

Am J Perinatol Rep 2016;6:e77-e82.

\begin{abstract}
Keywords

- gestational weight gain

- pre-pregnancy body mass index

- pregnancy outcomes

- obesity

Obesity during pregnancy and excessive weight gain during this period are associated with several maternal-fetal and neonatal complications. Moreover, a significant percentage of women have weight retention in the postpartum period, especially those with excessive weight gain during pregnancy. The recommendations of the 2009 Institute of Medicine were based on observational studies that have consistently shown that women with weight gain within the recommended range had better outcomes during pregnancy. In patients with obesity, however, there is no recommendation for weight gain, according to the class of obesity. This review, therefore, aims to evaluate the evidence on key maternal and fetal complications related to low weight gain during pregnancy in obese and overweight patients.
\end{abstract}

The prevalence of obesity has risen significantly worldwide in recent decades. Estimates from the National Health and Nutrition Examination Survey shows that approximately $70 \%$ of adult Americans are overweight, defined as body mass index (BMI) greater than or equal to $25 \mathrm{~kg} / \mathrm{m}^{2}$ or obese (BMI greater than or equal to $30 \mathrm{~kg} / \mathrm{m}^{2}$ ). ${ }^{1}$ Notably, about a quarter of women retain more than $4 \mathrm{~kg}$ after pregnancy. ${ }^{2}$ Pre-pregnancy weight and gestational weight gain (GWG) are important determinants of weight retention maintenance over the female lifespan. ${ }^{3}$

In 2009, the Institute of Medicine (IOM) ${ }^{4}$ updated GWG recommendations, taking into account the pre-pregnancy BMI categories defined by the World Health Organization (WHO) position and evidence from several cohort studies published since 1990. Lower GWG was recognized as safe for obese women, but the guideline did not specifically address the three obesity subclasses. ${ }^{5}$

In this review, we discuss aspects related to weight gain during pregnancy in women with obesity classes I, II, and III, emphasizing the effects of lower than recommended GWG on maternal and neonatal outcomes.

\section{Recommendations on Weight Gain during Pregnancy}

\section{Historical Overview}

The first recommendations on GWG were published in the 1970s and were developed to account for high rates of infant and childhood mortality. ${ }^{6}$ At that time, the positive association between lower GWG and low or very low birth weight had been recognized. For this reason, the American College of Obstetricians and Gynecologists (ACOG) recommended a total GWG of $10 \mathrm{~kg}$, as opposed to the usual practice, that consisted of recommending approximately $6.8 \mathrm{~kg}$ of GWG. ${ }^{5}$

Since then, GWG recommendations have been gradually increasing. In 1990, for the first time, the Nutrition During Pregnancy Committee advised that GWG should be set according to pre-pregnancy BMI, to optimize fetal growth received

July 15, 2015

accepted after revision

October 1, 2015

published online

November 2, 2015
DOI http://dx.doi.org/

10.1055/s-0035-1566309. ISSN 2157-6998.
Copyright $\odot 2016$ by Thieme Medical Publishers, Inc., 333 Seventh Avenue, New York, NY 10001, USA. Tel: +1(212) 584-4662.
License terms

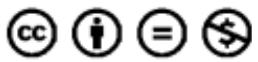


and pregnancy outcomes. ${ }^{7}$ Recommendations were based on Metropolitan Life Insurance weight-height tables. ${ }^{7}$ Despite the 1990 IOM recommendations on GWG, major changes in the demographic and epidemiological profiles of obesity in pregnancy occurred worldwide. The increasing obesity epidemic also affects reproductive age women. ${ }^{5}$ Several studies described remarkable GWG, far above recommendations, especially among overweight or obese women. ${ }^{8}$ It was estimated that $37 \%$ of normal pre-pregnancy BMI weight women, and $64 \%$ of overweight women, gained weight above the 1990 IOM recommendations. ${ }^{5}$ Hence, due to the association of both excessive GWG and higher pre-pregnancy BMI with poor maternal and fetal outcomes, a reassessment of criteria was carried in $2009 .^{5}$

\section{Current Recommendations}

The 2009 IOM guidelines development was based on BMI categories of the WHO. ${ }^{9}$ This report sets out specific GWG range for all obese women. ${ }^{5}$ These recommendations were endorsed by ACOG, ${ }^{10}$ and by the National Institute for Health and Care Excellence ${ }^{11}$ and are following described.

1. Underweight (pre-pregnancy BMI less than $18.5 \mathrm{~kg} / \mathrm{m}^{2}$ ): GWG between 12.5 and $18 \mathrm{~kg}$ (28 and $40 \mathrm{lb}$ )

2. Normal weight (pre-pregnancy BMI 18.5-24.9 kg/m²): GWG between 11.5 and $16 \mathrm{~kg}$ (25 and $35 \mathrm{lb}$ )

3. Overweight (pre-pregnancy BMI $25-29.9 \mathrm{~kg} / \mathrm{m}^{2}$ ): GWG between 7 and $11.5 \mathrm{~kg}$ (15 and $25 \mathrm{lb}$ )

4. Obesity (pre-pregnancy BMI at least $30 \mathrm{~kg} / \mathrm{m}^{2}$ ): GWG between 5 and $9 \mathrm{~kg}$ (11 and $20 \mathrm{lb}$ )

Current recommendations are based on observational studies that consistently showed improved pregnancy outcomes when GWG met the recommendations of the 2009 IOM position statement. ${ }^{5}$ Nevertheless, it should be noted that these recommendations were not adjusted for age, ethnicity, marital status, parity, and smoking.

The appropriate GWG for obese women, however, remains unclear. So far, there is no official recommendation stratified by obesity classes. Few studies examined the effects of different GWGs on obstetric outcomes in obese women and there is some evidence that low GWG and even weight loss could improve both maternal and fetal outcomes. ${ }^{12-14}$

Dietary interventions associated with a GWG as low as $3.84 \mathrm{~kg}$ were associated with decreased risk of preeclampsia and shoulder dystocia, and no effect on newborn weight. ${ }^{15} \mathrm{~A}$ recent meta-analysis of observational studies, most of them retrospective and including American cohorts, showed a reduction of about $20 \%$ in the risk of large for gestation age (LGA) with an increased risk of small for gestational age (SGA) between 17 and $24 \%$, with no risk gradient between the three classes of obesity. ${ }^{16}$

\section{Associated Factors}

Many hormonal and genetic factors are responsible for the variability in maternal metabolism during and after pregnancy. Leptin is correlated with the amount of adipose tissue, and its secretion is stimulated by insulin. Higher concentrations of leptin occur in the third trimester, concurrently to the physiological hyperinsulinemia characteristic of that period. Higher leptin levels were associated with greater GWG and postpartum weight retention in observational studies. ${ }^{17}$ Elevated levels of sex steroids, such as high progesterone, can affect insulin resistance which, in turn, could result in greater fat accumulation during pregnancy and postpartum weight retention. ${ }^{17}$

Several other factors such as ethnicity, age, marital status, socioeconomic status, cultural beliefs, smoking, and physical activity can contribute to the variability of $\mathrm{GWG}^{18}$ as described below. Longitudinal studies recorded an average GWG in African-descent women three to four times higher than that of Caucasian women after adjustment for prepregnancy weight. ${ }^{19,20}$

Pregnancy in adolescence has been associated with an increased risk of preterm birth, low birth weight, and neonatal mortality. ${ }^{21}$ Current recommendations suggest that pregnant adolescents should gain weight according to pre-pregnancy BMI, except for those aged 16 years or less whose menarche occurred within the past 2 years, due to the risk of compromising the final height. ${ }^{5}$ Conversely, in women over 35 years, a significantly higher risk of adverse outcomes during pregnancy, such as stillbirth, low birth weight, preterm birth and SGA, has been recorded. ${ }^{22,23}$ Moreover, older women also are at higher risk for hypertension, gestational diabetes mellitus (GDM), placenta previa, and placental abruption. ${ }^{24}$

Regular physical activity can modulate GWG. The results of a recent meta-analysis showed that women who engage in a program of aerobic or resistance training in the first or second trimesters (20 to 60 minutes three times a week) had a lower (610 g) weight gain compared with the control group, with no significant impact on fetal growth. ${ }^{25}$

\section{Effects of Low Gestational Weight Gain}

\section{Maternal Outcomes}

GDM, preeclampsia, cesarean or instrumental delivery, postpartum hemorrhage, and weight retention, are maternal adverse outcomes associated with excessive GWG and higher pre-pregnancy BMI. ${ }^{8,26}$ Studies evaluating GWG across different classes of obesity, found insufficient weight gain or even weight loss during pregnancy associated with a decreased risk of preeclampsia, postpartum hemorrhage, instrumental, and cesarean section, and postpartum weight retention. $^{13,27}$

\section{Preeclampsia}

There is a twofold risk of preeclampsia in overweight women and threefold in obese pregnant women ${ }^{28}$ showing a gradient proportional to the increase in BMI. ${ }^{29}$ Blomberg evaluated the effect of weight loss or insufficient GWG in more than 46,000 Swedish pregnancies stratified for different obesity classes. ${ }^{30}$ In this population-based cohort study, class III obese women who gained up to $4.9 \mathrm{~kg}$ during pregnancy had a lower risk of preeclampsia with unaffected risk of low Apgar score, SGA, and LGA, or fetal distress ${ }^{30}$ compared with same class obese women gaining weight within the 2009 IOM recommendations. 
However, there was a twofold increased risk of SGA among class III obese women who lost weight. Recent meta-analysis showed a reduced risk of $10 \%$ for preeclampsia in pregnant women with the GWG below the 2009 IOM guidelines. ${ }^{9}$

\section{Postpartum Hemorrhage}

A recent retrospective cohort study from the Consortium on Safe Labor, including 20,950 obese American women with a singleton term live birth, described maternal and neonatal outcomes according to weight change and BMI class. ${ }^{31}$ Low GWG (0-4.9 kg) or even weight loss were associated with a nonsignificant decrease in postpartum hemorrhage rates for women with class I to III obesity. Blomberg reported a significant increase in postpartum hemorrhage attributable to uterine atony at rates proportional to obesity class. ${ }^{12,30}$

\section{Cesarean Birth and Operative Vaginal Delivery}

Potential confounding factors to be considered when evaluating rates of cesarean birth associated with excess GWG are the route of previous delivery in multiparous women and the presence of comorbidities such as preeclampsia and GDM which most of the studies do not adjust as covariates. ${ }^{5}$

A prospective population-based Norwegian study showed a linear increased risk of cesarean birth, according to prepregnancy BMI after adjustments for parity. ${ }^{32}$ Recently, based on a retrospective, observational database from the Consortium on Safe Labor acquired from 12 institutions across 9 ACOG districts, the authors evaluated the probability of cesarean birth and operative vaginal delivery (forceps or vacuum) for each weight change category using the 2009 IOM recommendations. ${ }^{31}$ The predicted probability of cesarean birth increased linearly as weight increased for all obesity classes, whereas operative vaginal delivery did not vary significantly with weight change. When stratified by parity, multiparous class III obese women with low GWG (range, 0-4.9 kg) had a 33\% reduced risk of cesarean birth, whereas no effect for multiparous class I and class II obese women was observed. Blomberg et al showed that classes I to III obese women who lost weight during pregnancy had a lower risk (range, 24-34\%) of cesarean birth. ${ }^{13}$

\section{Postpartum Weight Retention}

Most studies described weight retention between 0.4 and $3.8 \mathrm{~kg}$ after pregnancy, with different lengths of follow-up, extended up to 156 months after delivery. ${ }^{33,34}$ GWG seems to be the most important predictor for postpartum weight retention. $^{33}$ A recent meta-analysis of nine observational studies classified women, according to the 2009 IOM criteria and found that, compared with women with adequate GWG, those with a lower GWG had significantly less postpartum weight retention, an average of $-2.99 \mathrm{~kg}$ at short and medium interval follow-up. ${ }^{35}$ Another meta-analysis suggested that women who exceeded GWG have a long-term trend of greater postpartum weight retention with an increased risk of overweight or obesity at 21 years of follow-up. ${ }^{36}$ Nevertheless, the contribution of socioeconomic factors, cultural practices, lifestyle changes, breastfeeding, and other related behaviors for postpartum weight retention must be elucidated.

\section{Fetal, Neonatal, and Childhood Outcomes}

Maternal weight gain above the 2009 IOM recommendations has been strongly associated with an increased birth weight, later obesity, and its metabolic consequences throughout life. ${ }^{5}$ However, a lower GWG among obese women seems to reduce outcomes such as fetal distress, low Apgar score, LGA, although the effect on risk of SGA is controversial.

\section{Fetal Growth}

Birth weight related to gestational age is an important determinant of sibling outcomes. Newborns are classified as adequate for gestational age (AGA), SGA (weight for gestational age below the 10th percentile) or LGA (weight for gestational age above the 90th percentile). Macrosomia is usually defined as a term birth weight above 4,000 or 4,500 g.

Obese women who lost weight during pregnancy had a lower absolute risk of the LGA, but higher absolute risk of SGA. $^{27,28}$ For obese women, the proportion of SGA can increase with every $1 \mathrm{~kg}$ weight loss during pregnancy. ${ }^{37}$ Adequate ranges of GWG associated with minimal risks of LGA and SGA can differ across pre-pregnancy BMI groups in this high-risk population.

Blomberg et al assessed the effects of lower GWG and weight loss during pregnancy on fetal growth. ${ }^{30}$ Compared with the limited GWG of 5 to $9 \mathrm{~kg}$, women with class I obesity who lost weight had a higher risk of SGA, while a gain of 0.1 to $4.9 \mathrm{~kg}$ was not related to increased risk of SGA, LGA, and macrosomia. Bodnar et al took the 2009 IOM recommendations as reference and found that a GWG of $2.2 \mathrm{~kg}$ to less than $5 \mathrm{~kg}$ for class III obese women was associated with a probability of less than 10\% LGA and SGA births. ${ }^{14}$ Hinkle et al evaluated the risk for SGA, LGA, and macrosomia and suggested that a GWG below the 2009 IOM guidelines could be associated with more adequate birth weight. ${ }^{15}$

A pilot study evaluated GWG retrospectively in patients with obesity and type 2 diabetes mellitus in relation to fetal growth and perinatal morbidity. ${ }^{38}$ Patients with the GWG below $5 \mathrm{~kg}$ (mean, $3.7 \mathrm{~kg}$ ) had lower rates of the LGA, preterm birth, and perinatal morbidity compared with those with an average GWG of $12 \mathrm{~kg}$, after adjusting for pre-pregnancy BMI. Catalano et al suggested that weight loss or even a gain of less than $5 \mathrm{~kg}$ in overweight or obese women were associated with increased rates of SGA, with a negative effect on anthropometric birth measures. ${ }^{39}$ However, results were not stratified according to obesity class. Data from a recent systematic review showed no consensus about the increased risk of SGA along with the different classes of obesity, and a decreased risk of LGA in obese women with a lower GWG. ${ }^{28}$ It should be mentioned that analyses were performed with a very small number of studies. ${ }^{28}$

\section{Other Fetal Outcomes}

Preterm birth, fetal distress, Apgar score, shoulder dystocia, congenital anomalies, and childhood obesity have not yet been fully evaluated or were not evaluated at all regarding the 2009 IOM recommendations.

Preterm birth, defined as before 37 weeks completed gestation, is a critical indicator of fetal maturity and is directly 
associated with the risk of mortality and morbidity according to the grade of prematurity. ${ }^{5}$ In obese women, an association of lower GWG with preterm birth remains uncertain. A longitudinal cohort study in Massachusetts, Project Viva, investigated the rate of GWG associated with the lowest combined risk of five short- and long-term maternal and child health outcomes (preterm birth, maternal postpartum weight retention, SGA, LGA, and child obesity at age 3 years) for 2,012 mother-child pairs recruited between 1999 and $2002 .^{40}$ The lowest predicted outcome prevalence occurred with a $0.19 \mathrm{~kg} / \mathrm{wk}$ loss for pregnant women with a prepregnancy $\mathrm{BMI} \geq 30.0 \mathrm{~kg} / \mathrm{m}^{2} .40$

Fetal distress was evaluated in a few studies of classes I to III obese women with low GWG or weight loss. It seems reasonably safe for obese women to gain a minimal weight with no increased risk for most maternal and fetal adverse outcomes. $^{13}$

A low Apgar score, defined as less than 7 at 5 minutes, was evaluated in only two observational studies. ${ }^{13,31}$ Newborns of classes I to III obese women with low GWG (0-5 kg) did not markedly differ from newborns of obese women who gained weight according to the 2009 IOM recommendations.

Maternal obesity appears to be associated with a small but independent increased risk for fetal malformations including neural tube defects (NTD), cardiac anomalies, orofacial clefts, hydrocephaly, limb reduction anomalies as well as stillbirth and macrosomia. ${ }^{41}$ Mechanisms linking congenital malformations to maternal obesity are not known, but they could be related to an altered intrauterine nutritional milieu as well as to hyperinsulinemia. ${ }^{42}$ Moreover, the protective effect of folic acid supplementation in reducing the risk of NTD may not be observed in obese women. ${ }^{41}$ Unfortunately, prenatal screening of malformations is significantly limited because maternal obesity can lessen the ultrasound detection rate of fetal anomalies by at least $20 \%$ compared with women with a normal BMI. ${ }^{43}$

Several observational studies have described an association between GWG above the 2009 IOM recommendations and greater adiposity in the offspring. ${ }^{44}$ Preliminary results suggest that a low GWG in obese women could attenuate childhood adiposity-related outcomes of the offspring. Recently, a retrospective study described the association of GWG with offspring outcomes at an average age of 10.4 years. $^{44}$ The effect of maternal pre-pregnancy BMI on several childhood outcomes was attenuated in the offspring of mothers with adequate versus excessive GWG. A suboptimal GWG conveys no benefit or risk on children's overweight and abdominal adiposity as described in a retrospective cohort study in Germany that investigated the interrelationship between inadequate or excessive GWG, according to maternal pre-pregnancy BMI. ${ }^{45}$

\section{Effect of Weight Loss during Pregnancy}

Weight loss during pregnancy can have short- and long-term repercussions for both mother and fetus. Weight loss may be accompanied by ketonuria, whose effect on fetal and child neurodevelopment remains uncertain. To assess whether prolonged fasting (13-18 h/d) during the month of Ramadan could cause ketonemia or ketonuria with a significant impact on intrauterine fetal development, a prospective study was conducted in Turkey. ${ }^{46} \mathrm{~A}$ total of 36 patients above 20 weeks of gestation of an uneventful pregnancy fasted for periods of 18 days on an average. At delivery, there were significant differences neither in ultrasonographic fetal measures or estimated fetal weight, nor in maternal serum ketone bodies when compared with nonfasting pregnant controls.

So far, no randomized weight loss trials in obese pregnant women are available. Observational studies favor the idea of weight loss in obese patients, as it seems to reduce some obesity-related complications during pregnancy at the expense of a potentially higher risk of an SGA. ${ }^{31}$ A retrospective analysis of more than 122,000 pregnancies in Atlanta suggests that a weight loss up to $5 \mathrm{~kg}$ in class II and III obese women, conferred a favorable risk-benefit profile without an increased risk of SGA. ${ }^{15}$ As previously cited, Blomberg et al found a reduction in the rates of cesarean birth, preeclampsia, and LGA, offset by an increased risk of SGA in class II and III obese patients who lost weight during pregnancy. ${ }^{13}$ However, the authors argued that the twofold increase in risk of SGA birth $(3.7 \%)$ in class III obese women who lost weight is only slightly above the overall population prevalence in Sweden (3.6\%). More recently, Kominiarek et al reported a risk for SGA infants in classes I to III obese women who lost an average 4.8 to $5.6 \mathrm{~kg}$ of weight when compared with women with the GWG between 5 and $9 \mathrm{~kg} .{ }^{31}$ The lowest average predicted probability of adverse outcomes (cesarean birth, postpartum hemorrhage, SGA, LGA, neonatal care unit admission) occurred when classes I to III obese pregnant women lost weight (up to $9.1 \mathrm{~kg}$ ).

\section{Perspectives: Areas of Uncertainty}

Mechanisms by which body weight influences reproductive function and performance are not well understood and need further clarification. ${ }^{47}$ The effects of maternal BMI status, insulin resistance, and inflammation on placental function and birth outcomes need to be examined in greater detail. ${ }^{47}$ Furthermore, there is a great need for research on the relationships of amount, composition, and pattern of GWG for overweight, obese, and severely obese women related to short- and long-term health outcomes for mother and child. ${ }^{47}$

It is not known whether a high maternal pre-pregnancy BMI contributes to an intrauterine developmental programming process that increases the risk of childhood overweight and long-term disease, or whether the obesogenic environment of home and family is the primary determinant of childhood obesity and subsequent diseases. ${ }^{47}$ Hence, studies assessing the epigenetic mechanisms that underline and/or potentiate the effects of GWG on fetal outcomes are needed. ${ }^{5}$ It is important to consider that current recommendations for GWG are from American cohorts whose offspring's weight was AGA. These recommendations have not been adjusted for variables such as age, parity, smoking, and ethnicity. Therefore, it is not yet known which optimal GWG is recommended according to variables other than maternal pre-pregnancy BMI. 


\section{Conclusion}

Evidences from observational studies after the publication of the 2009 IOM guidelines suggest that a lower GWG (range, 0-5 kg) could influence LGA and SGA risks in opposite directions, with decreased risk for LGA and increased risk for SGA mainly in obesity classes I to II. It is important to emphasize that each obese BMI class consists of a distinct population with different magnitudes of baseline and pregnancy-related risks. Furthermore, weight management should be primarily based on fetal growth, since this outcome was the most widely assessed in all studies.

Validation of results from observational studies must come from trials with adequate statistical power to answer whether dietary intervention as well as physical activity to induce lower GWG in obese women will reduce deleterious maternal and fetal outcomes in this population.

\section{Conflict of Interest}

The authors declare no conflict of interest.

\section{References}

1 Hedley AA, Ogden CL, Johnson CL, Carroll MD, Curtin LR, Flegal KM. Prevalence of overweight and obesity among US children, adolescents, and adults, 1999-2002. JAMA 2004;291(23):2847-2850

2 Kac G, Benício MH, Velásquez-Meléndez G, Valente JG, Struchiner CJ. Gestational weight gain and prepregnancy weight influence postpartum weight retention in a cohort of brazilian women. J Nutr 2004;134(3):661-666

3 Phelan S. Pregnancy: a "teachable moment" for weight control and obesity prevention. Am J Obstet Gynecol 2010;202(2):135.e1-135. e8

4 Nucci LB, Schmidt MI, Duncan BB, Fuchs SC, Fleck ET, Santos Britto MM. Nutritional status of pregnant women: prevalence and associated pregnancy outcomes. Rev Saude Publica 2001;35(6): 502-507

5 Rasmussen KM, Yaktine AL. Institute of Medicine and National Research Council Committee to Reexamine IOM Pregnancy Weight Guidelines. Weight Gain during Pregnancy: Reexamining the Guidelines. Washington (DC): National Academies Press (US); 2009

6 Maternal Nutrition and the Course of Pregnancy. Washington (DC): NRC (National Research Council); 1970

7 Institute of Medicine (IOM). Nutrition during Pregnancy: Part I, Weight Gain: Part II, Nutrient Supplements. Washington (DC): National Academies Press (US); 1990

8 Johnson J, Clifton RG, Roberts JM, et al; Eunice Kennedy Shriver National Institute of Child Health; Human Development (NICHD) Maternal-Fetal Medicine Units (MFMU) Network. Pregnancy outcomes with weight gain above or below the 2009 Institute of Medicine guidelines. Obstet Gynecol 2013;121(5):969-975

9 National Institutes of Health. Clinical Guidelines on the Identification, Evaluation, and Treatment of Overweight and Obesity in Adults-The Evidence Report. Obes Res 1998;6(Suppl 2):51S-209S

10 American College of Obstetricians and Gynecologists. ACOG Committee opinion no. 549: obesity in pregnancy. Obstet Gynecol 2013;121(1):213-217

11 NICE (National Institute for Health and Care Excellence). Weight management before, during and after pregnancy. NICE guidelines [PH 27]. Published date: July 2010. Available at: guidance.nice.org. uk/ph27. Accessed October 25, 2015
12 Blomberg M. Maternal and neonatal outcomes among obese women with weight gain below the new Institute of Medicine recommendations. Obstet Gynecol 2011;117(5):1065-1070

13 Bodnar LM, Siega-Riz AM, Simhan HN, Himes KP, Abrams B. Severe obesity, gestational weight gain, and adverse birth outcomes. Am J Clin Nutr 2010;91(6):1642-1648

14 Hinkle SN, Sharma AJ, Dietz PM. Gestational weight gain in obese mothers and associations with fetal growth. Am J Clin Nutr 2010; 92(3):644-651

15 Thangaratinam S, Rogozinska E, Jolly K, et al. Effects of interventions in pregnancy on maternal weight and obstetric outcomes: meta-analysis of randomised evidence. BMJ 2012;344:e2088

16 Kapadia MZ, Park CK, Beyene J, Giglia L, Maxwell C, McDonald SD. Can we safely recommend gestational weight gain below the 2009 guidelines in obese women? A systematic review and metaanalysis. Obes Rev 2015;16(3):189-206

17 Stein TP, Scholl TO, Schluter MD, Schroeder CM. Plasma leptin influences gestational weight gain and postpartum weight retention. Am J Clin Nutr 1998;68(6):1236-1240

18 Beyerlein A, Schiessl B, Lack N, von Kries R. Optimal gestational weight gain ranges for the avoidance of adverse birth weight outcomes: a novel approach. Am J Clin Nutr 2009;90(6): 1552-1558

19 Keppel KG, Taffel SM. Pregnancy-related weight gain and retention: implications of the 1990 Institute of Medicine guidelines. Am J Public Health 1993;83(8):1100-1103

20 Smith DE, Lewis CE, Caveny JL, Perkins LL, Burke GL, Bild DE. Longitudinal changes in adiposity associated with pregnancy. The CARDIA Study. Coronary Artery Risk Development in Young Adults Study. JAMA 1994;271(22):1747-1751

21 Chen XK, Wen SW, Fleming N, Demissie K, Rhoads GG, Walker M. Teenage pregnancy and adverse birth outcomes: a large population based retrospective cohort study. Int J Epidemiol 2007;36(2): 368-373

22 Delpisheh A, Brabin L, Attia E, Brabin BJ. Pregnancy late in life: a hospital-based study of birth outcomes. J Womens Health (Larchmt) 2008;17(6):965-970

23 Endres J, Dunning S, Poon SW, Welch P, Duncan H. Older pregnant women and adolescents: nutrition data after enrollment in WIC. J Am Diet Assoc 1987;87(8):1011-1016, 1019

24 Joseph KS, Allen AC, Dodds L, Turner LA, Scott H, Liston R. The perinatal effects of delayed childbearing. Obstet Gynecol 2005; 105(6):1410-1418

25 Streuling I, Beyerlein A, von Kries R. Can gestational weight gain be modified by increasing physical activity and diet counseling? A meta-analysis of interventional trials. Am J Clin Nutr 2010;92(4): 678-687

26 Macdonald-Wallis C, Tilling K, Fraser A, Nelson SM, Lawlor DA. Gestational weight gain as a risk factor for hypertensive disorders of pregnancy. Am J Obstet Gynecol 2013;209(4):327.e1-327.e17

27 Kiel DW, Dodson EA, Artal R, Boehmer TK, Leet TL. Gestational weight gain and pregnancy outcomes in obese women: how much is enough? Obstet Gynecol 2007;110(4):752-758

28 Catalano PM. Increasing maternal obesity and weight gain during pregnancy: the obstetric problems of plentitude. Obstet Gynecol 2007;110(4):743-744

29 Bodnar LM, Catov JM, Klebanoff MA, Ness RB, Roberts JM. Prepregnancy body mass index and the occurrence of severe hypertensive disorders of pregnancy. Epidemiology 2007;18(2): 234-239

30 Blomberg M. Maternal obesity and risk of postpartum hemorrhage. Obstet Gynecol 2011;118(3):561-568

31 Kominiarek MA, Seligman NS, Dolin C, et al. Gestational weight gain and obesity: is 20 pounds too much? Am J Obstet Gynecol 2013;209(3):214.e1-214.e11

32 Morken NH, Klungsøyr K, Magnus P, Skjærven R. Pre-pregnant body mass index, gestational weight gain and the risk of operative delivery. Acta Obstet Gynecol Scand 2013;92(7):809-815 
33 Linné Y, Dye L, Barkeling B, Rössner S. Long-term weight development in women: a 15-year follow-up of the effects of pregnancy. Obes Res 2004;12(7):1166-1178

34 Linné Y, Barkeling B, Rössner S. Long-term weight development after pregnancy. Obes Rev 2002;3(2):75-83

35 Nehring I, Schmoll S, Beyerlein A, Hauner H, von Kries R. Gestational weight gain and long-term postpartum weight retention: a meta-analysis. Am J Clin Nutr 2011;94(5):1225-1231

36 Mannan M, Doi SA, Mamun AA. Association between weight gain during pregnancy and postpartum weight retention and obesity: a bias-adjusted meta-analysis. Nutr Rev 2013;71(6):343-352

37 Park S, Sappenfield WM, Bish C, Salihu H, Goodman D, Bensyl DM. Assessment of the Institute of Medicine recommendations for weight gain during pregnancy: Florida, 2004-2007. Matern Child Health J 2011;15(3):289-301

38 Asbjörnsdóttir B, Rasmussen SS, Kelstrup L, Damm P, Mathiesen ER. Impact of restricted maternal weight gain on fetal growth and perinatal morbidity in obese women with type 2 diabetes. Diabetes Care 2013;36(5):1102-1106

39 Catalano PM, Mele L, Landon MB, et al; Eunice Kennedy Shriver National Institute of Child Health and Human Development Maternal-Fetal Medicine Units Network. Inadequate weight gain in overweight and obese pregnant women: what is the effect on fetal growth? Am J Obstet Gynecol 2014;211(2):137.e1-137.e7

40 Oken E, Kleinman KP, Belfort MB, Hammitt JK, Gillman MW. Associations of gestational weight gain with short- and longer- term maternal and child health outcomes. Am J Epidemiol 2009; 170(2):173-180

41 Stothard KJ, Tennant PW, Bell R, Rankin J. Maternal overweight and obesity and the risk of congenital anomalies: a systematic review and meta-analysis. JAMA 2009;301(6):636-650

42 Weintraub AY, Levy A, Levi I, Mazor M, Wiznitzer A, Sheiner E. Effect of bariatric surgery on pregnancy outcome. Int J Gynaecol Obstet 2008;103(3):246-251

43 Best KE, Tennant PW, Bell R, Rankin J. Impact of maternal body mass index on the antenatal detection of congenital anomalies. BJOG 2012;119(12):1503-1511

44 Kaar JL, Crume T, Brinton JT, Bischoff KJ, McDuffie R, Dabelea D. Maternal obesity, gestational weight gain, and offspring adiposity: the exploring perinatal outcomes among children study. J Pediatr 2014;165(3):509-515

45 Ensenauer R, Chmitorz A, Riedel C, et al. Effects of suboptimal or excessive gestational weight gain on childhood overweight and abdominal adiposity: results from a retrospective cohort study. Int J Obes 2013;37(4):505-512

46 Dikensoy E, Balat O, Cebesoy B, Ozkur A, Cicek H, Can G. Effect of fasting during Ramadan on fetal development and maternal health. J Obstet Gynaecol Res 2008;34(4):494-498

47 Siega-Riz AM, King JC; American Dietetic Association; American Society of Nutrition. Position of the American Dietetic Association and American Society for Nutrition: obesity, reproduction, and pregnancy outcomes. J Am Diet Assoc 2009;109(5):918-927 